\title{
FOURIER-STIELTJES TRANSFORMS WHICH VANISH AT INFINITY OFF CERTAIN SETS
}

\author{
by LOUIS PIGNO
}

(Received 29 June, 1976)

0. Introduction. In this paper $G$ is a nondiscrete compact abelian group with character group $\Gamma$ and $M(G)$ the usual convolution algebra of Borel measures on $G$. We designate the following subspaces of $M(G)$ employing the customary notations: $M_{a}(G)$ those measures which are absolutely continuous with respect to Haar measure; $M_{s}(G)$ the space of measures concentrated on sets of Haar measure zero and $M_{d}(G)$ the discrete measures.

The Fourier-Stieltjes transform of the measure $\mu \in M(G)$ is defined by

$$
\hat{\mu}(\gamma)=\int_{G} \gamma(-x) d \mu(x) \quad(\gamma \in \Gamma) .
$$

The ideal of measures whose transforms vanish at infinity will be denoted by $M_{0}(G)$.

Let $L^{p}(G)(1 \leq p \leq \infty)$ be the Lebesgue space of index $p$ formed with respect to Haar measure on $G$ and $C(G)$ those $f \in L^{\infty}(G)$ which are continuous. For any subspace $B(G)$ of $M(G)$ and subset $E$ of $\Gamma$ put

$$
B_{E}(G)=\{\mu \in B(G): \hat{\mu}=0 \text { off } E\} .
$$

In section 1 we give several extensions of a classical theorem due to Rajchman [23] which we state for the circle group $\mathbf{T}$ as

THEOREM 1. Suppose $\mu \in M(\mathbf{T})$ and $\hat{\mu}(n)=o(1)$ for all $n>0$ or all $n<0$. Then $\mu \in M_{0}(\mathbf{T})$.

An analogue of Theorem 1 is valid for any compact abelian group with ordered dual in the sense of relating the behavior of a Fourier-Stieltjes transform at " $+\infty$ " with its

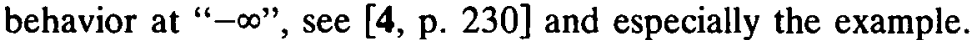

A subset $\Re$ of $\Gamma$ will be called a Rajchman set if whenever $\mu \in M(G)$ and $\hat{\mu} \in$ $C_{0}(\Gamma \backslash \Re)$, then $\mu \in M_{0}(G)$. Here $C_{0}(\Gamma \backslash \mathfrak{R})$ means those complex-valued functions on $\Gamma$ which vanish at infinity off $\Re$. In section 1 we prove the following two theorems which we now cite.

(i) The union of a Rajchman set and a Sidon set is a Rajchman set;

(ii) The union of a Rajchman set and any set $\mathscr{E}$ satisfying $\left.\left.M_{a}(G)^{\wedge}\right|_{\delta} \subset M_{d}(G)^{\wedge}\right|_{\delta}$ is a Rajchman set.

Let $E$ be a Rider set contained in $\mathbb{Z}^{+}$, the positive part of $\mathbb{Z}$. We also prove in section 1 that:

(iii) $\mathbb{Z}^{-} \cup E^{n}$ is a Rajchman set.

In section 2 we present some examples of Rajchman sets in $\Gamma$. The sum sets in example (III) are especially interesting from an arithmetic point of view. In section 3 we 
exhibit a connection between Rajchman sets in the integer group $\mathbb{Z}$ and idempotent measures on the circle. The result of this section provided the original motivation for our study of Rajchman sets.

1. Union problems for Rajchman sets. A subset $S$ of $\Gamma$ is said to be a Sidon set if whenever $f \in L_{\mathbf{S}}^{\infty}(G)$, then $\sum|\hat{f}(\boldsymbol{\alpha})|<\infty$. Our first result is:

THEOREM 2. The union of a Rajchman set and a Sidon set is a Rajchman set.

Proof. We adapt the proof of [5]. Let $\hat{\mu} \in C_{0}(\Gamma \backslash \Re \cup S)$, where $\Re$ is Rajchman and $\mathbf{S}$ is Sidon. By a result of Drury, given a natural number $m$ there is a measure $\nu_{m} \in M(G)$ such that

$$
\begin{array}{cc}
\hat{\nu}_{m}(\alpha)=1 & (\alpha \in \mathbf{S}), \\
\left|\hat{\nu}_{m}(\alpha)\right|<\frac{1}{m} \quad(\alpha \notin \mathbf{S}) .
\end{array}
$$

Since $\mathfrak{R}$ is Rajchman it follows from (1) that the measure

$$
\mu-\nu_{m} * \mu \in M_{0}(G) .
$$

On letting $m \rightarrow \infty$ in (3) we gather from (2) that $\hat{\mu} \in C_{0}(\Gamma \backslash \mathbf{S})$. Our result now follows from Theorem 2.20 of $[16$, p. 30].

We remark that Theorem 2.20 of [16, p. 30] shows that the method of proof of [5] works for any compact abelian group to establish that the union of a small $p$ set and a Sidon set is a small $2 p$ set. For if $\Gamma$ is not ordered or the positive cone is not Rajchman then the first part of the argument of [5] shows that $\hat{\mu} \in C_{0}(\Gamma \backslash \mathbf{S})$. An improvement of [5] due to the author and S. Saeki can be found in [22, p. 91]. The reader is also referred to example I of the present paper.

Next, let $\mathscr{E}$ satisfy

$$
\left.\left.M_{a}(G)^{\wedge}\right|_{\&} \subset M_{d}(G)^{\wedge}\right|_{\&} .
$$

The interpolation property $(*)$ has been studied by many authors; the reader is referred to [2], [11], [14], and [21]. It is known that the set of prime powers and the sets $\left\{r^{n}+r^{m}: n, m \in \mathbb{Z}^{+}\right\}$where $r \in \mathbb{Z}^{+}$and $r \geq 2$ satisfy $(*)$ for $\Gamma=\mathbb{Z}$; see [10].

THEOREM 3. The union of a Rajchman set $\mathfrak{R}$ and any set $\mathscr{E}$ satisfying (*) is a Rajchman set.

Proof. Let $\mu \in M(G)$ and suppose

$$
\hat{\mu} \in C_{0}(\Gamma \backslash \mathscr{E} \cup \Re) .
$$

Let $\alpha_{0} \in \mathscr{E}$ and choose $\nu \in M_{a}(G)$ such that

$$
\hat{\nu}\left(\alpha_{0}\right)=1 .
$$

Since $\mathscr{E}$ satisfies $(*)$ we gather there is a measure $\mu_{d} \in M_{d}(G)$ such that

$$
\hat{\mu}_{d}=\hat{\nu} \text { on } \mathscr{E} \text {. }
$$


By (1)

$$
\left(\mu_{d} * \mu-\nu * \mu\right)^{\wedge} \in C_{0}(\Gamma \backslash \mathfrak{R})
$$

thus

$$
\mu_{d} * \mu-\nu * \mu \in M_{0}(G)
$$

In light of the Riemann-Lebesgue Lemma, (4) implies that

$$
\mu_{d} * \mu \in M_{0}(G)
$$

Put

$$
M_{0}^{\perp}(G)=\left\{\rho \in M(G): \rho \text { is singular with each } \tau \in M_{0}(G)\right\} .
$$

Then, as is well known, $M(G)=M_{0}(G) \oplus M_{0}^{\perp}(G)$. Since $M_{0}(G)$ is translation invariant and since $M_{0}^{\perp}(G)$ is translation invariant and closed, it follows that if $\rho_{d} \in M_{d}(G)$, then

$$
\rho_{d} * M_{0}^{\perp}(G) \subset M_{0}^{\perp}(G)
$$

Write $\mu=\mu_{0}+\mu_{\perp}$ where $\mu_{0} \in M_{0}(G)$ and $\mu_{\perp} \in M_{0}^{\perp}(G)$. We infer from (5) that $\mu_{d} * \mu_{\perp} \in M_{0}(G)$, so we obtain via (6) that

$$
\mu_{d} * \mu_{\perp}=0 \text {. }
$$

But (2) and (3) in combination with (7) yield $\left(\mu_{\perp}\right)^{\wedge}\left(\alpha_{0}\right)=0$. Thus

$$
\left(\mu * \mu_{\perp}\right)^{\wedge} \in C_{0}(\Gamma \backslash \Re)
$$

and so $\mu * \mu_{\perp} \in M_{0}(G)$. It is now evident that $\mu_{\perp} * \mu_{\perp} \in M_{0}(G)$ and this is possible if and only if $\mu_{\perp}=0$.

Theorem 3 implies the following result which we state without proof.

Every non-Sidon subset of a discrete abelian group contains a Rajchman set which is non-Sidon.

For the remainder of this section our notation will be for the most part that of $[\mathbf{1 6}]$. In what follows $\Gamma$ is ordered by the positive cone $\mathscr{P}$.

A subset $S$ of $\Gamma$ is called asymmetric if $0 \notin S$ and $\alpha \in S$ imply $-\alpha \notin S$. For any subset $E$ of $\Gamma$ and integer $s \geq 0, R_{s}(E)$ denotes the number of asymmetric subsets $S$ of $E \cup-E$ satisfying $|S|=s$ and $\sum_{\alpha \in S} \alpha=0$. The set $E$ is called a Rider set if there is a constant $B>0$ such that $R_{s}(E) \leq B^{s}$ for all $s$. For $k \in \mathbb{Z}^{+}$let $E_{k}$ consist of all characters of the form $\sum_{\alpha \in S} \alpha$ where $S$ is an asymmetric subset of $E \cup-E$ and $|S|=k$.

Before presenting our result we shall need the next two propositions, which we now state for the readers' convenience.

Proposition 1. (Bonami [3]). Let $E$ be a Rider set in $\Gamma$. Then $E_{k}$ is a $\Lambda(q)$ set for all $1 \leq q<\infty$ and all $k>0$. 
Recall that a set $A$ is called a $\Lambda(q)$ set if there exists a $p<q$ and a constant $K_{p}$ such that

$$
\|t\|_{q}<K_{p}\|t\|_{p}
$$

for all trigonometric polynomials $t$ on $G$ with $\hat{t}=0$ off $A$.

Proposmion 2. (Rudin [24]). Let $\mu \in M(\mathbf{T})$ and $A$ a set of type $\Lambda(1)$. If supp $\hat{\mu} \subset$ $\mathbb{Z}^{-} \cup A$, then $\mu \in M_{a}(\mathbf{T})$.

For a generalization of Proposition 2 see [18]. In this connection we point out that for the corollary of $[18, p .369]$ to be valid we must replace the word infinity by " $-\infty$ " in both the statement of the corollary and its proof. We shall call a subset $R$ of $\Gamma$ a weak Rajchman set if whenever supp $\hat{\mu} \subset R$ then $\mu \in M_{0}(G)$. The set of Proposition 2 is a weak Rajchman set which is (to the author's knowledge) not known to be Rajchman. The method of Theorem 2 shows that the union of a weak Rajchman set and a Sidon set is a weak Rajchman set.

We shall be interested in showing that for certain $\Lambda(1)$ sets $A$ (which are not Sidon) $\mathbb{Z}^{-} \cup A$ is a Rajchman set. For any subset $E$ of $\Gamma$ and natural number $n \geq 2$ put

$$
E^{n}=\left\{n_{j_{1}}+n_{j_{2}}+\ldots+n_{j_{n}}: n_{j_{1}} \neq n_{j_{k}}, n_{j_{1}} \in E\right\}
$$

and $E^{1}=E$. We now present this extension of Rajchman's Theorem:

THeOREM 4. Let $E$ be a Rider set in $\mathbb{Z}$ such that $E \subset \mathbb{Z}^{+}$. Then $\mathbb{Z}^{-} \cup E^{n}$ is a Rajchman set.

Proof. Let $\mu \in M(T)$ and $\hat{\mu} \in C_{0}\left(\mathbb{Z} \backslash \mathbb{Z}^{-} \cup E^{n}\right)$. We shall suppose $\hat{\mu} \notin C_{0}(\mathbb{Z})$ and force a contradiction.

If $\hat{\mu} \notin C_{0}(\mathbb{Z})$ then there exists by Theorem 1 a $\delta>0$ such that the set

$$
\mathscr{S}=\left\{\alpha \in E^{n}:|\hat{\mu}(\alpha)| \geq \delta\right\}
$$

is infinite. Choose a sequence $\left\langle\alpha_{j}\right\rangle_{1}^{\infty}$ with the $\alpha$ 's distinct and in $\mathscr{S}$.

Let $\nu$ be any weak-star cluster point of $\left\{-\alpha_{j} \mu\right\}$. Since the $\alpha$ 's are distinct it follows from the Helson Translation Lemma that

$$
\nu \in M_{s}(\mathbf{T})
$$

Observe that

$$
\hat{\nu}(0) \neq 0
$$

since the $\alpha$ 's belong to $\mathscr{S}$.

We shall fix $\alpha \in \mathbb{Z}^{+}$and calculate $\hat{\nu}(\alpha)$. If $\alpha+\alpha_{i}$ meets $E^{n}$ only finitely many times then, since $\hat{\mu} \in C_{0}\left(\mathbb{Z} \backslash \mathbb{Z}^{-} \cup E^{n}\right)$, it follows that $\hat{\nu}(\alpha)=0$. Thus if $\alpha \in \mathbb{Z}^{+}$, then $\hat{\nu}(\alpha) \neq 0$ implies that

$$
\alpha \in \varlimsup_{j}\left(E^{n}-\alpha_{i}\right)=\bigcap_{m=1}^{\infty} \bigcup_{k=m}^{\infty}\left(E^{n}-\alpha_{k}\right)
$$


Well, it is easy to see that

where

$$
\bigcup_{j=1}^{\infty}\left(E^{n}-\alpha_{i}\right) \subset A,
$$

$$
A=\{0\} \cup E_{2} \cup E_{4} \ldots \cup E_{2 n} \quad(n \geq 1) .
$$

By Proposition 1 and [23, p. 217] this set $A$ is a $\Lambda(q)$ set for all finite $q$. Inasmuch as $\nu$ is singular and supp $\hat{\nu} \subset \mathbb{Z}^{-} \cup A$ we obtain via Proposition 2:

$$
\hat{\nu}(0)=0 \text {. }
$$

Since (4) contradicts (2) we conclude that $\hat{\mu} \dot{\in} C_{0}(\mathbb{Z})$; i.e. $\mu \in M_{0}(\mathbf{T})$ and this finishes our proof.

COROLlary. If $E$ and $F$ are disjoint with $E \cup F$ dissociate and $E \cup F \subset \mathbb{Z}^{+}$, then $\mathbb{Z}^{-} \cup(E+F)$ is a Rajchman set.

For some interesting properties of $E+F$ where $E$ and $F$ are disjoint with dissociate union see [1].

2. Examples of Rajchman Sets. In this section we present some examples of Rajchman sets in $\Gamma$. Most of the examples appear in the literature implicitly.

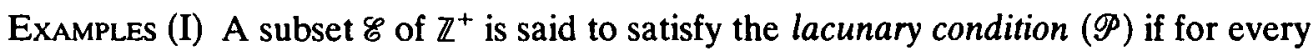
increasing sequence $n_{1}, n_{2}, \ldots, \in E, \mathbb{Z}^{+} \cap \varliminf_{\left(\mathscr{E}-n_{j}\right)}$ is a finite set.

Then if $\mathscr{E}$ satisfies $(\mathscr{P}), \mathbb{Z}^{-} \cup \mathscr{E}$ is a Rajchman set. To confirm this assertion it suffices to repeat the proof of Theorem 3 of [8]. A proof for arbitrary discrete $\Gamma$ that a Sidon set is a Rajchman set can be based on Theorem 1.4 of $[16$, p. 8] and the method of proof of the present example.

(II) A subset $\mathscr{F}$ of $\mathbb{Z}$ is said to be a set of uniform convergence or $U C$-set if every $f \in C_{\mathscr{F}}(\mathbf{T})$ has uniformly convergent Fourier series. Non-Sidon $U C$-sets were first exhibited by Figà-Talamanca; see [16, pp. 82-86]. Careful scrutiny of the proof of Theorem 5 of [7] shows that $\mathbb{Z}^{-} \cup \mathscr{F}$ is a Rajchman set.

(III) A subset $\mathbb{E}$ of $\mathbb{Z}$ is defined to be a strong Rajchman set if $\mathbb{E}$ is a Rajchman set. Here $\vec{E}$ is a closure of $\mathbb{E}$ in $\mathbb{Z}$, where $\mathbb{Z}$ has the relative topology of its Bohr compactification, $\overline{\mathbb{Z}}$. A subset $\mathscr{R}$ of $\mathbb{Z}$ is said to be a Riesz set if $M_{\mathscr{R}}(\mathbf{T})=L_{\mathfrak{R}}^{1}(\mathbf{T})$. A subset $\mathscr{E}$ of $\mathbb{Z}$ is called a strong Riesz set if $\overline{\mathscr{E}}$ is a Riesz set.

Replacing the decomposition $M(\mathbf{T})=M_{a}(\mathbf{T}) \oplus M_{s}(\mathbf{T})$ by $M_{0}(\mathbf{T}) \oplus M_{0}^{\perp}(\mathbf{T})$ in [17] and adapting the methods of Meyer we can easily prove: If $\mathfrak{A}$ is a Rajchman set and $\mathbb{E}$ is a strong Rajchman set then $\Re \cup \in$ is a Rajchman set. In particular we have that the union of a Rajchman set and a strong Riesz set is a Rajchman set. The following examples of strong Riesz sets can be found in [6] and [17] respectively:

(i) The set of integers expressible as the sum of two perfect squares is a strong Riesz set; all $k$.

(ii) Let $n_{k}$ be a sequence of positive integers such that $n_{k+1} / n_{k}$ is an integer $>2$ for 
Then the set of all finite sums of the form

$$
\sum_{k \geq 0} t_{k} n_{k} \quad \text { with } \quad t_{k} \in\{0,1\}, \quad n_{i} \neq n_{j}
$$

is a strong Riesz set.

Here is another example due to the author and S. Saeki:

Fix any two sequences $\left\langle p_{n}\right\rangle_{1}^{\infty}$ and $\left\langle q_{n}\right\rangle_{1}^{\infty}$ of natural numbers $\geq 2$. Let

$$
\mathscr{E}_{n}=\left\{p_{1} p_{2} \ldots p_{n} k: k=0, \pm 1, \ldots, \pm q_{n}\right\}
$$

and put $\mathscr{E}=\bigcup_{1}^{\infty} \mathscr{E}_{n}$. Then $\mathscr{E}+\ldots+\mathscr{E}$ (any finite number of summands) is a strong Riesz set.

To see this let

$$
\mathbf{D}=\left\{e^{2 \pi i m / p_{1} \cdots p_{n}}: m \in \mathbb{Z} \text { and } n \in \mathbb{Z}^{+}\right\} .
$$

Consider $\mathbf{D}$ with the discrete topology and $\hat{\mathbf{D}}$ the compact dual of $\mathbf{D}$. It is easy to prove that the only accumulation point of $\mathscr{E}^{+}$in $\hat{\mathbf{D}}$ is 0 ; see $[13$, p. 107] and $[13$, p. 403]. Since $\hat{\mathbf{D}}$ is a factor group of $\overline{\mathbb{Z}}$ and $\mathbf{D}$ is dense in $\mathbf{T}$ we gather that the set of accumulation points of $\mathscr{E}^{+}$in $\mathbb{Z}$ (with the relative Bohr topology) is a subset of $\{0\}$. Thus $\mathscr{E}^{+}$is strong Riesz and moreover since $\mathscr{E}$ is symmetric we have that $\mathscr{E}^{-}$is strong Riesz. Thus $\mathscr{E}=\mathscr{E}^{+} \cup \mathscr{E}^{-}$is strong Riesz.

Now the set of accumulation points for $(\mathscr{E}+\mathscr{E})^{+}$in $\hat{\mathbf{D}}$ is a subset of $\{0\} \cup \mathscr{E}$. So since $\mathscr{E}$ is strong Riesz it follows from $[\mathbf{1 7}$, p. 90$]$ that $(\mathscr{E}+\mathscr{E})^{+}$is strong Riesz. Thus $\mathscr{E}+\mathscr{E}=$ $(\mathscr{E}+\mathscr{E})^{+} \cup(\mathscr{E}+\mathscr{E})^{-}$is strong Riesz and the proof for any finite number of summands follows inductively.

(IV) Let $\mathfrak{R}$ be a Rajchman set in $\Gamma$. Suppose $\mathfrak{A} \subset \Gamma$ has the property that

$$
\{(\mathfrak{U}-\alpha) \cap \mathfrak{A}\} \text { is a Rajchman set }
$$

for all $\alpha \notin \Re$. Then $\mathfrak{A}$ is a Rajchman set.

The proof of the above statement is similar to the proof of Theorem 2 of $[19, p .77]$ and we omit the details.

3. Weak Rajchman sets and idempotent measures. Using a remarkable result of $\mathrm{K}$. de Leeuw and Y. Katznelson, we establish a connection between idempotent measures and Rajchman sets.

THEOREM 5. Suppose $R$ is a weak Rajchman set in $\mathbb{Z}$ and $\mu \in M(\mathbf{T})$ such that $\hat{\mu}=\hat{\mu}^{2}$ off $R$. Then there is an idempotent measure $\nu \in M(\mathbf{T})$ such that $\hat{\mu}=\hat{\nu}$ off $R$.

Proof. Let $\mu \in M(\mathbf{T})$ with

$$
\hat{\mu}=\hat{\mu}^{2} \quad \text { off } \quad R \text {. }
$$

Via (1)

$$
\limsup _{n \notin R}\left|\hat{\mu}(n)-\hat{\mu}^{2}(n)\right|=0
$$


and since $R$ is weak Rajchman, we gather that

$$
\limsup _{n \in \mathbf{Z}}\left|\hat{\mu}(n)-\hat{\mu}^{2}(n)\right|=0 .
$$

As a consequence of Theorem 2 of $[4$, pp. 220-221] (2) gives:

$$
\mu=\mu_{1}+\mu_{2}, \quad \hat{\mu}_{1} \text { periodic with } \mu_{1} \text { idempotent }
$$

and

$$
\limsup _{n \in \mathbf{Z}}\left|\hat{\mu}_{2}(n)\right|=0 \text {. }
$$

As a consequence of (1), (3), and (4), we deduce that $\hat{\mu}=\hat{\mu}_{1}$ off $R \backslash F$ where $F$ is some finite set. Thus, since $\hat{\mu}_{1}$ is periodic, we are done.

Theorem 5 is an extension of a theorem of $\mathrm{H}$. Helson [12]; see also [15] and [20]. We conclude our paper with some open questions.

(i) If $\mathscr{R}$ is a Riesz set must $\mathscr{R}$ be a Rajchman set?

(ii) Is the union of a Rajchman set and a $U C$-set a Rajchman set? In this connection see example (II) of section 2.

A subset $\mathbf{A} \subset \Gamma$ is called a Rosenthal set if $L_{\mathbf{A}}^{\infty}(G)=C_{\mathbf{A}}(G)$. It is known that the sum sets $\mathscr{E}+\ldots+\mathscr{E}$ in example (III) of section 2 are Rosenthal sets. The following question suggests itself:

(iii) Is the union of a Rajchman set and a Rosenthal set a Rajchman set? An analogous result can be found in [9].

After our manuscript had been accepted for publication the author generalized Theorem 5 to compact abelian groups.

Note added in proof. The author has recently learned of the work of Keiji Izuchi, Sidon sets and small $M_{0}$-sets, Sci. Rep. Tokyo Kyoiku Daigaku Sect. A 12 (1974), 146-148. Izuchi proves Theorem 2 of the present paper using the method of [5]. Theorem 2 was also known to the author in 1974.

\section{REFERENCES}

1. R. C. Blei, A tensor approach to interpolation phenomena in discrete abelian groups, Proc. Amer. Math. Soc. 49 (1975), 175-177. 211-214.

2. R. C. Blei, On Fourier-Stieltjes transforms of discrete measures, Math. Scand. 35 (1974),

3. A. Bonami, Etude des coefficients de Fourier des fonctions de $L^{p}(G)$, Ann. Inst. Fourier (Grenoble) 20 (1970), 335-402.

4. K. de Leeuw and Y. Katznelson, The two sides of a Fourier-Stieltjes transform and almost idempotent measures, Israel J. Math. 8 (1970), 213-229.

5. R. E. Dressler, W. Parker and L. Pigno, Sidon sets and small $p$ sets, Quart, J. Math. Oxford Ser. 2, 24 (1973), 79-80.

6. R. E. Dressler and L. Pigno, On strong Riesz sets, Colloq. Math. 29 (1974), 157-158.

7. R. E. Dressler and L. Pigno, Sets of uniform convergence and strong Riesz sets, Math. Ann. 211 (1974), 227-231. 
8. R. E. Dressler and L. Pigno, Some lacunary conditions for Fourier-Stieltjes transforms, Ark. Mat. 13 (1975), 73-77.

9. R. E. Dressler and L. Pigno, Une remarque sur les ensembles de Rosenthal et Riesz, C.R. Acad. Sci. Paris, 280 (1975), 280-281.

10. R. E. Dressler and L. Pigno, Modification sets and transforms of discrete measures, Acta. Sci. Math. (Szeged) 38 (1976), 13-16.

11. S. Hartman and C. Ryll-Nardzewski, Almost periodic extensions of functions, II, Colloq. Math. 15 (1966), 79-86.

12. H. Helson, On a theorem of Szegö, Proc. Amer. Math. Soc. 6 (1955), 235-242.

13. E. Hewitt and K. A. Ross, Abstract Harmonic Analysis, Vol. 1 (Springer Verlag, 1963).

14. J.-P. Kahane, Ensembles de Ryll-Nardjewski et ensembles de Helson, Colloq. Math. 15 (1966), 87-92.

15. I. Kessler, Semi-idempotent measures on abelian groups, Bull. Amer. Math. Soc. 73 (1967), $258-260$.

16. J. M. López and K. A. Ross, Sidon Sets (Marcel Dekker, Inc., New York, 1975).

17. Y. Meyer, Spectres des mesures et mesures absolument continues, Studia Math. 30 (1968), 87-99.

18. L. Pigno, A variant of the F. and M. Riesz theorem, J. London Math. Soc. (2), 9 (1974), 368-370.

19. L. Pigno, Convolution products with small Fourier-Stieltjes transforms, Illinois J. Math. 19 (1975), 77-78.

20. L. Pigno, Integer-valued Fourier-Stieltjes transforms, Technical Report No. 53 Kansas State University (1976).

21. L. Pigno and S. Saeki, Interpolation by transforms of discrete measures, Proc. Amer. Math. Soc. 52 (1975), 156-158.

22. L. Pigno and S. Saeki, Fourier-Stieltjes Transform which vanish at infinity, Math. Z. 141 (1975), 83-91.

23. A. Rajchman, Une classe de series trigonometriques ... Math. Ann. 101 (1929), 686-700.

24. W. Rudin, Trigonometric series with gaps, J. Math. and Mech. 9 (1970), 203-228.

KANSAS STATE UNIVERSITY

ManhatTan

KANSAS 66506, U.S.A. 